\title{
Erratum to: Effect of vegetation type, wetting intensity, and nitrogen supply on external carbon stimulated heterotrophic respiration and microbial biomass carbon in forest soils
}

\author{
WU HaoHao ${ }^{1,2}$, XU XingKai $^{1 *}$, DUAN CunTao ${ }^{1}$, LI TuanSheng $^{3} \&$ CHENG WeiGuo $^{4}$ \\ ${ }^{1}$ State Key Laboratory of Atmospheric Boundary Layer Physics and Atmospheric Chemistry, Institute of Atmospheric Physics, \\ Chinese Academy of Sciences, Beijing 100029, China; \\ ${ }^{2}$ College of Earth Science, University of Chinese Academy of Sciences, Beijing 100049, China; \\ ${ }^{3}$ College of Earth Science and Resources, Chang'an University, Xi'an 710054, China; \\ ${ }^{4}$ Faculty of Agriculture, Yamagata University, Tsuruoka 997-8555, Japan
}
Citation: Wu H H, Xu X K, Duan C T, Li T S, Cheng W G. 2017. Erratum to: Effect of vegetation type, wetting intensity, and nitrogen supply on external carbon stimulated heterotrophic respiration and microbial biomass carbon in forest soils. Science China Earth Sciences, 60: 1382, doi: $10.1007 / \mathrm{s} 11430-017-9049-6$

\begin{abstract}
Erratum to: SCIENCE CHINA Earth Sciences, August 2015 Vol.58 No.8: 1446-1456 doi:10.1007/s11430-015-5058-x
\end{abstract}

The data of TOC, TN and Water-extractable DON in Table 1 should be changed to those in bold as follows:

Table 1 Main soil properties under the both temperate forests ${ }^{\text {a) }}$

\begin{tabular}{|c|c|c|c|c|c|c|c|c|c|c|c|c|c|c|c|}
\hline \multirow{2}{*}{$\begin{array}{l}\text { Vegetation } \\
\text { types }\end{array}$} & \multirow{2}{*}{$\begin{array}{l}\text { Moisture } \\
(\%, w / w)\end{array}$} & \multirow{2}{*}{$\begin{array}{c}\mathrm{pH} \\
\text { (water) }\end{array}$} & \multirow{2}{*}{$\begin{array}{c}\text { TOC } \\
\left(\mathrm{mg} \mathrm{C} \mathrm{g}^{-1}\right)\end{array}$} & \multirow{2}{*}{$\begin{array}{c}\mathrm{TN} \\
\left(\mathrm{mg} \mathrm{N} \mathrm{g}^{-1}\right)\end{array}$} & \multicolumn{4}{|c|}{$\begin{array}{c}\text { Water-extractable } \mathrm{C} \text { and } \mathrm{N} \text { pools } \\
\left(\mathrm{mg} \mathrm{C} \text { or } \mathrm{N} \mathrm{kg}^{-1}\right)\end{array}$} & \multicolumn{4}{|c|}{$\begin{array}{c}\mathrm{K}_{2} \mathrm{SO}_{4} \text {-extractable } \mathrm{C} \text { and } \mathrm{N} \text { pools } \\
\left(\mathrm{mg} \mathrm{C} \text { or } \mathrm{N} \mathrm{kg}^{-1}\right)\end{array}$} & \multirow{2}{*}{$\begin{array}{c}\mathrm{MBN} \\
\left(\mathrm{mg} \mathrm{N}^{-1}\right. \\
\left.\mathrm{kg}^{-1}\right)\end{array}$} & \multirow{2}{*}{$\begin{array}{c}\mathrm{MBC} \\
(\mathrm{mg} \mathrm{C} \\
\left.\mathrm{kg}^{-1}\right)\end{array}$} & \multirow{2}{*}{$\begin{array}{l}\text { MBC:MBN } \\
\quad \text { ratio }\end{array}$} \\
\hline & & & & & $\mathrm{NO}_{3}^{-}-\mathrm{N}$ & $\mathrm{NH}_{4}^{+}-\mathrm{N}$ & DON & DOC & $\mathrm{NO}_{3}^{-}-\mathrm{N}$ & $\mathrm{NH}_{4}^{+}-\mathrm{N}$ & DON & DOC & & & \\
\hline WBF & 31.7 & 5.67 & 56.8 & 4.5 & 20.2 & 10.4 & 6.2 & 87.2 & 17.7 & 20.7 & 26.9 & 176.6 & 312 & 1985 & 6.3 \\
\hline BKPF & 50.7 & 5.87 & 82.6 & 7.1 & 51.7 & 4.2 & 8.4 & 69.7 & 39.4 & 4.8 & 28.2 & 167.7 & 226 & 1716 & 7.7 \\
\hline
\end{tabular}

The online version of the original research paper can be found at http://link.springer.com/article/10.1007/s11430015-5058-x. 